Net hod for est i mat ing the frequency- wavenumber resolved power spectrumdensi ty usi ng the maxi mum ent ropy met hod for I i mited spat i al poi nt $\mathrm{s}$

\begin{tabular}{|l|l|}
\hline $\begin{array}{l}\text { jour nal or } \\
\text { publ i cat } \mathrm{i} \text { on } \mathrm{ti} \mathrm{tl} \text { e }\end{array}$ & $\mathrm{Pl}$ asma Physi cs and Cont rol I ed Fusi on \\
\hline vol une & 63 \\
\hline number & 4 \\
\hline page $\mathrm{r}$ ange & 045011 \\
\hline year & $2021-02-19$ \\
\hline URL & ht t p: //hdl . handl e. net /10655/00012586 \\
\hline
\end{tabular}




\title{
Method for estimating the frequency-wavenumber resolved power spectrum density using the maximum entropy method for limited spatial points
}

\author{
T. Kobayashi ${ }^{1,2}$, T. Nishizawa ${ }^{3}$, M. Sasaki ${ }^{4}$, M. Yoshinuma ${ }^{1}$, and \\ K. Ida ${ }^{1}$ \\ ${ }^{1}$ National Institute for Fusion Science, National Institutes of Natural Sciences, Toki 509-5292, \\ Japan \\ 2 The Graduate University for Advanced Studies, SOKENDAI, Toki 509-5292, Japan \\ ${ }^{3}$ Max Planck Institute for Plasma Physics, Boltzmannstr. 2, 85748 Garching, Germany \\ ${ }^{4}$ Research Institute for Applied Mechanics, Kyushu University, Kasuga 816-8580, Japan \\ E-mail: kobayashi.tatsuya@nifs.ac.jp
}

\begin{abstract}
Combination of the Fourier transform and the maximum entropy method for estimating the frequency-wavenumber resolved power spectrum density is proposed. After illustrating the physical insight of the maximum entropy method by using synthetic test data, capability of the proposed method is tested using numerical simulation data. The method is also applied to experimental data obtained by the beam emission spectroscopy in the Large Helical Device. All of those examinations show that the proposed method provides more plausible results than conventional methods when the available spatial points are limited.
\end{abstract}

\section{Introduction}

The frequency-wavenumber resolved power spectrum density is a powerful analysis tool widely used in the magnetically confined plasma community. Such an analysis 
is often performed for the poloidal or azimuthal direction in low temperature basic plasma devices by using the Fourier transform (FT) $[1,2,3,4,5,6,7]$. This analysis allows the direct comparison between the experimental observation and the dispersion relation theoretically predicted. A poloidal or azimuthal probe array that covers the full circumference $[8,9,10]$ plays a crucial role for realizing the spatial FT with the periodic boundary condition. Availability of such a probe array is an advantage of basic devices. Even in high temperature torus devices, the frequency-wavenumber resolved power spectrum density is calculated as well with a limited coverage of the diagnostics by using the spatial FT $[11,12,13]$.

An alternative way to compute the frequency-wavenumber resolved spectrum is the so-called two-point statistical method [14]. Due to its applicability in a wide variety of situations, the two-point statistical method is frequently used for various occasions both in linear plasma columns $[15,16,17]$ and in torus devices $[18,19,20]$. However, this method is in principle inapplicable for two or more wave components at a frequency range, for instance, the standing wave [21]. In such a case, at least three probe tips are required to resolve the amplitude and the wavenumber of the constituents. A long discharge duration is also necessary for performing ensemble averaging to achieve the statistical convergence.

In a condition where a multiple channel fluctuation diagnostic that partly covers a particular spatial range is available, which is often realized in fusion devices through the beam emission spectroscopy, the Doppler back scattering, and others, a different 
path to reach the frequency-wavenumber resolved power spectrum density is possible by using the maximum entropy method (MEM) [22, 23, 24, 25, 26, 27]. MEM can estimate the power spectrum density with a very high resolution even when the input wave is truncated in a part of one cycle $[24,25]$. MEM is occasionally applied to fusion plasma data as well, aiming for improving the resolution in the wavenumber estimation with a limited number of measurement points $[11,28,29,30,31,32,33]$. In this paper, we systematically document a method for estimating the frequency-wavenumber resolved power spectrum density by applying FT to the time domain and MEM to the spatial domain. In section 2, the analysis principle of MEM is described by contrasting the difference between FT with a window function and MEM on a set of test data. Here, the physical insight into MEM is focused upon, therefore readers are recommended to refer to specialized literatures (e.g., $[24,25]$ ) for the comprehensive review of mathematical backgrounds. Section 3 introduces the two-dimensional FT-MEM method for estimating the frequency-wavenumber resolved power spectrum density. Application of the proposed method is presented in sections 4 and 5, to a numerical fluid turbulence simulation data and to a multichannel beam emission spectroscopy (BES) data in the Large Helical Device (LHD), respectively. A summary is given in section 6 .

\section{Comparison of Fourier transform and maximum entropy method}

In this section, FT and MEM are compared in estimating the power spectrum density of a time-dependent test signal. The power spectrum density $S(f)$ as a function of the 
frequency $f$ is defined by the Wiener-Khinchin $(\mathrm{W}-\mathrm{K})$ theorem,

$$
S(f)=\frac{1}{2 \pi} \int_{-\infty}^{\infty} C(\tau) e^{-i 2 \pi f \tau} d \tau
$$

where $C(\tau)$ is the autocorrelation function, which depends on the time delay $\tau$, and $i$ is the imaginary unit. In practice, any recorded experimental data have their finite data length, therefore the integral in Eq. (1) cannot be rigorously performed. Instead, the power spectrum density is estimated by giving certain assumptions for data outside the record length.

The most commonly used method for estimating the power spectrum density is FT via the fast $\mathrm{FT}(\mathrm{FFT})$ algorithm. In using FT for the power spectrum density estimation, the data with a finite length is assumed to repeat itself outside the record length. This assumption is reasonable for the data which are actually periodic, for example for the spatial distribution of a physical quantity in the poloidal or the toroidal direction in torus plasmas. However, in the time domain, the data is usually not periodic, therefore discontinuities appear at both ends of the data. In order to avoid this problem, various window functions, such as the Hanning window or the Tukey window, are often used. Applying a window function on the data reduced the relative importance of the data points near both ends. To compensate this effect, an overlapping moving average of the power spectrum density is performed for data with a long record length. However, FT with a window function does not necessarily perform well when the number of data points is limited, which is often the case for spatially sampled data.

A completely different methodology called MEM was introduced by Burg [22] in 
1967 for estimating the power spectrum density particularly from data with a limited record length. MEM estimates the power spectrum density by extrapolating the autocorrelation function to an infinite lag in such a way that the entropy density is maximized. The entropy density of a fluctuation data in a stationary Gaussian process is defined as

$$
h \propto \int_{-f_{\mathrm{N}}}^{f_{\mathrm{N}}} \log S(f) d f=\int_{-f_{\mathrm{N}}}^{f_{\mathrm{N}}} \log \left[\frac{1}{2 \pi} \int_{-\infty}^{\infty} C(\tau) e^{-i 2 \pi f \tau} d \tau\right] d f
$$

where $f_{\mathrm{N}}$ is the Nyquist frequency. On the one hand, maximizing the entropy density holding consistency of $S(f)$ in the recorded portion is regarded as a variational problem that can be directly solved by the method of Lagrange multiplier [24, 25]. On the other hand, maximizing the entropy density in extrapolating the autocorrelation function corresponds to maintaining the maximal ambiguity for the unavailable information but being consistent with the known information [24, 25, 27]. This extrapolation is equivalent to the linear prediction (LP) by autoregressive model fitting for the raw data, i.e.,

$$
\hat{x}_{k}^{(M)}=\sum_{j=1}^{M} a_{j} x_{k-j}
$$

where $\hat{x}_{k}^{(M)}$ is the linearly predicted value of a time series $x_{k}, a_{j}$ is the LP coefficients, and $M$ is the number of data points used for LP. The MEM spectrum estimate is given by the LP coefficients $a_{j}$ as

$$
S_{\mathrm{MEM}}^{(M)}(f)=\frac{\Delta t P_{M}}{\left|1-\sum_{j=1}^{M-1} a_{j} \exp (-i 2 \pi f j \Delta t)\right|^{2}},
$$


where $\Delta t$ is the data sampling time and $P_{M}$ is the variance of the estimation error, i.e.,

$$
P_{M}=N^{-1} \sum_{k=1}^{N}\left|x_{k}-\hat{x}_{k}^{(M)}\right|^{2} .
$$

The LP coefficients $a_{j}$ are obtained so as to minimize $P_{M}$, which is equivalent to maximizing the entropy density.

The windowed FT and MEM are compared using test data. The test data is defined as a simple cosine wave, $\cos (2 \pi f t)$, where $f=10.5$ and $t$ continues infinitely. For the data analysis, 100 points is taken with 0.01 sampling interval and labeled as $t=0$ to 0.99. At the sampling, the white Gaussian noise with the standard deviation of 0.2 is superposed. In order to perform ensemble averaging, 100 datasets are created. One of the test data series is shown in Fig. 1 (a). Results of the windowed FT with three different window functions (rectangular, Hanning, and Tukey) and MEM with the data points for LP of $M=9$ are displayed in Fig. 1 (b). The frequency resolution of MEM, which can be arbitrarily set, is $0.1 \mathrm{~Hz}$. MEM provides the power spectrum density that is sharpest and closest to the true spectrum, i.e., the Dirac delta function of $S(f)=\delta(f-10.5)$. Meanwhile the power spectrum densities obtained by the windowed FTs show peaks broadened to some extent. In particular, FT with the rectangular window gives a very wide tail much above the noise floor at $O\left(\sim 10^{-5}\right)$ likely due to the discontinuity of the repeated data as discussed below. The power spectrum density with the Tukey window appears with two sidebands at both sides of the peak. The result with the Hanning window is better than the other two window function cases, but still shows a substantial peak width. 

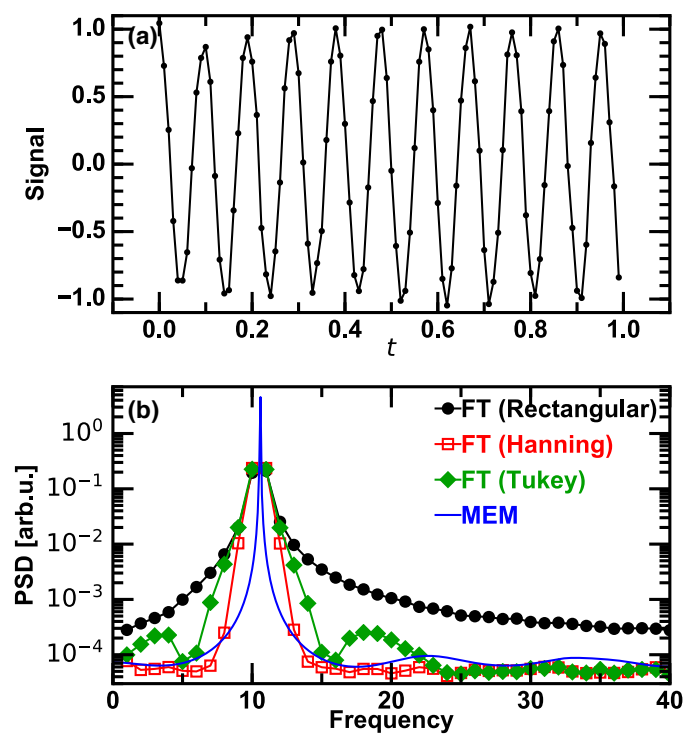

Figure 1. (a) Test data and (b) its power spectrum densities obtained by the Fourier transform with various window functions and the maximum entropy method.

Calculating the autocorrelation function from the obtained power spectrum densities provides a much clearer insight into the difference between the windowed FT and MEM. The autocorrelation function is given by

$$
C(\tau) \sim \int_{-f_{\mathrm{N}}}^{f_{\mathrm{N}}} S(f) e^{i 2 \pi f \tau} d f
$$

where the approximately equal sign is due to the integration truncated at the Nyquist frequency $f_{\mathrm{N}}$. Figures $2(\mathrm{a}-\mathrm{d})$ correspond to the autocorrelation functions obtained using Eq. (6) with various values of $\tau$. An envelope modulation with the period of $\tau=1$ is seen in all the autocorrelation functions for FT. In contrast, MEM provides an almost complete cosine wave with a constant amplitude of unity. The fluctuation frequency is consistent with the test data frequency of $f=10.5$, as expected of the $\mathrm{W}-\mathrm{K}$ theorem.

In order to discuss how the autocorrelation function is modulated for the FT cases and why not for the MEM case, the autocorrelation function is directly calculated from 

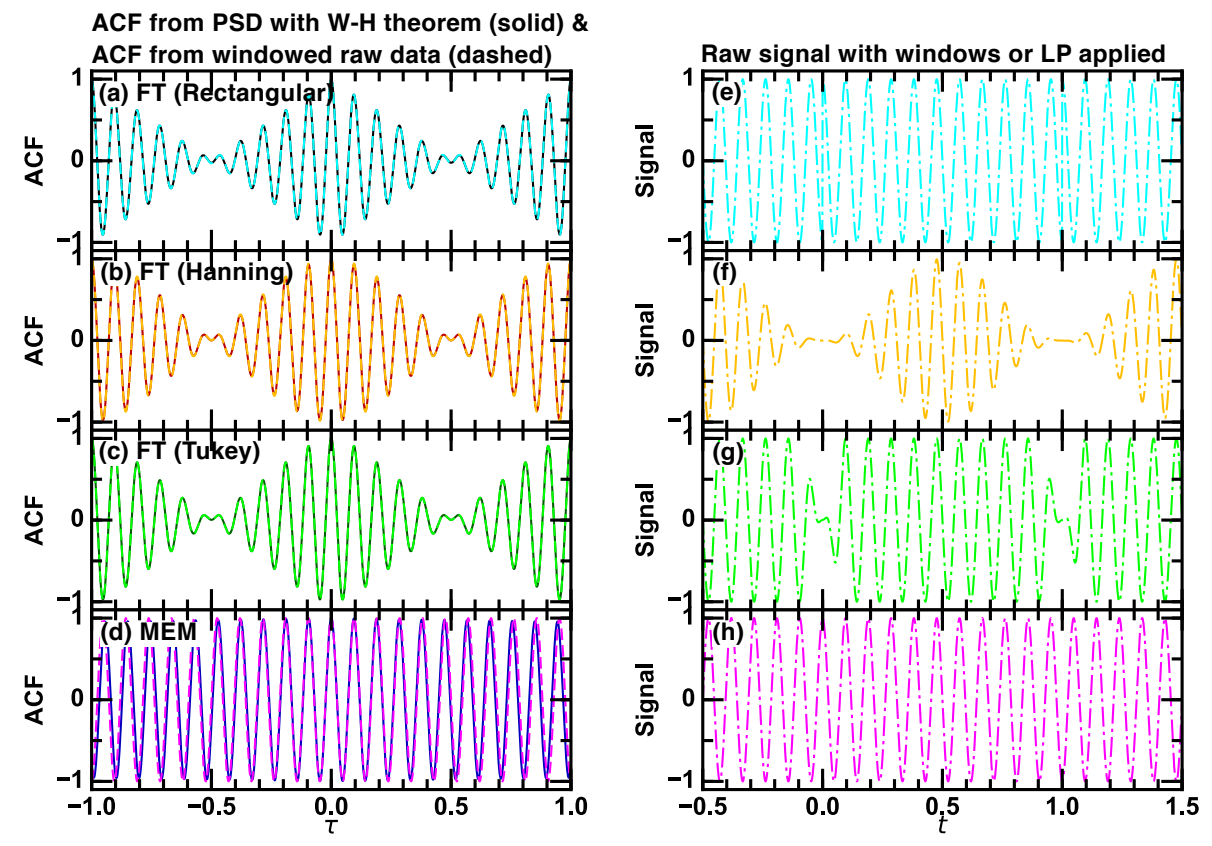

Figure 2. (a-d) Autocorrelation functions obtained by power spectrum densities in Fig. 1 (b) through the Wiener-Khinchin (W-K) theorem, (e-g) repeated raw data multiplied by window functions (rectangular, Hanning, and Tukey, respectively), and (h) raw data extrapolated by the linear prediction (LP). Autocorrelation functions directly obtained by data in (e-h) are overlaid in (a-d) by dashed curves.

the test data in the time domain. Figure 2 (e) is the repeated test data with the rectangular window function, i.e., no window function. Here, no statistical noise is applied to the data for simplicity. There are discontinuous points at $t=0$ and 1 , since the test cosine wave and the sampled data length are not synchronizing. The period of discontinuous jumps in the wave phase corresponds to the lifetime of this wave, which causes the envelope modulation in the autocorrelation function. Applying the window functions removes discontinuous points as shown in Figs. 2 (f) and (g), but in return modulates the data amplitude. The autocorrelation functions directly calculated from 
the data in Figs. 2 (e-g) are overlaid on Figs. 2 (a-c), which almost completely agree with those obtained via Eq. (6). The finite lifetime of the wave is reflected to the spectral peak width shown in Fig. 1 (b), where the half widths at the half maximum of the peaks are almost identical to the windowed FT results, although the tail shapes are very different. Note that the phase difference between two curves in Fig. 2 (d) gradually increases as $|\tau|$ increases. This is because the peak frequency of the MEM spectrum has $1 \%$ error.

Input for the direct calculation of the autocorrelation function in the MEM case is the raw data extrapolated by the LP scheme, which is plotted in Fig. 2 (h). Here, $M=9$ is again used as the number of points for LP. By LP, the almost complete cosine wave is reproduced for $t<0$ and $t>1$, which therefore maximizes the entropy density as a result. LP extends the lifetime of the data extremely long, which is reflected in the sharp spectral peak of MEM as shown in Fig. 1 (b). The autocorrelation function directly calculated from the extended raw data is overlaid on that obtained via Eq. (6), showing a good agreement here as well.

In the actual application of MEM, the result is sometimes sensitive to the choice of the parameter $M$. Too large value of $M$ can cause an overfitting in the estimated power spectrum density. There is a scheme to systematically specify the most appropriate value of $M$ according to the Akaike final prediction error (FPE) criterion [34, 24, 25]. The value of $M$ for the current example is chosen based on this scheme, which has provided plausible results. However, the $M$ value provided by the Akaike FPE criterion 
is not necessarily always most reasonable in practice, and a heuristic determination of $M$ is occasionally inevitable. In contrast, FT is stable in most of the situations. Therefore, if there is no specific need in using MEM, e.g., the limitation of the data points, it is safe to use FT.

\section{Two-dimensional FT-MEM for the frequency-wavenumber spectral decomposition}

The frequency-wavenumber resolved power spectrum density is calculated by applying FT and MEM to data in the time-space domain. The analysis procedure is shown in Fig. 3. First, FT is applied in the time domain to signals at each channel to compute the complex Fourier component as a function of the frequency. The complex MEM [26] is then operated in the space domain for each frequency, providing the complex frequency-wavenumber resolved FT-MEM component $X(f, k)$. The power spectrum density is calculated as $\left\langle X(f, k) X(f, k)^{*}\right\rangle$, where $*$ and \langle\rangle denote the complex conjugate and the ensemble averaging, respectively. As $X(f, k)$ is a complex quantity, the cross spectrum or the cross coherence can also be calculated, although they are out of the scope in this paper. See Appendix for an example of the python code for the complex MEM calculation. 

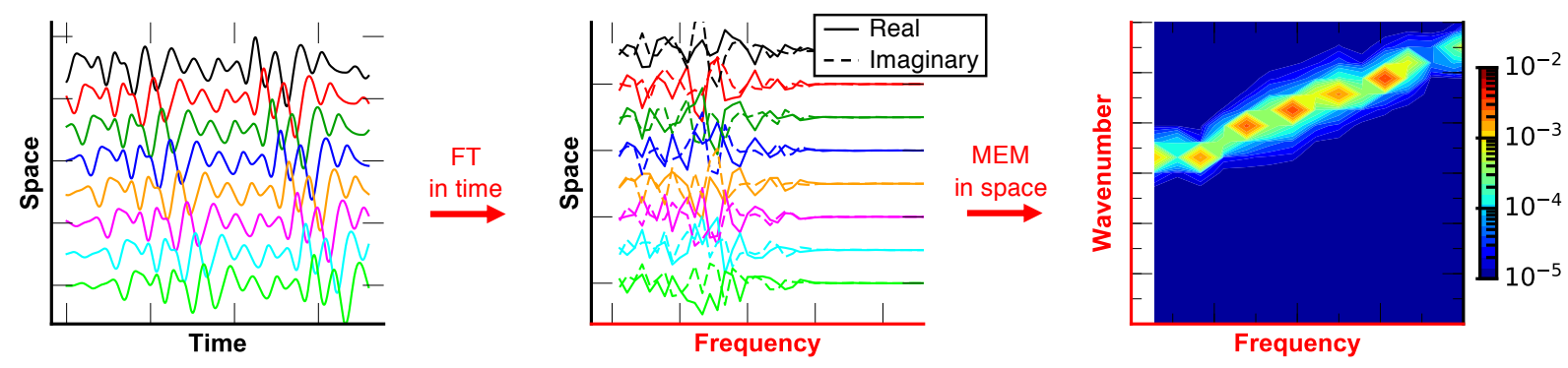

Figure 3. Analysis procedure of the two-dimensional FT-MEM for the frequency-wavenumber spectral decomposition.

\section{Application I: Numerical simulation data}

The proposed analysis method is applied to the results of a numerical simulation for the cylindrical plasma turbulence performed in the Numerical Linear Device (NLD) [35]. The simulation code provides the three dimensional distribution of plasma quantities, including in the azimuthal direction (the poloidal direction for the torus geometry) distribution. By using the entire azimuthal circumference for the spatial spectrum estimation, the true power spectrum density is obtained since the cyclic boundary condition is naturally satisfied. Meanwhile, by limiting the available azimuthal angle, the experimental situation is simulated and the capability of the FT-MEM (for the time-space domain) method is evaluated by contrasting that of the conventional FT-FT method and the two-point estimation [14].

The model for the numerical simulation is the global three-field Hasegawa-Wakatani reduced fluid model for the electron density, the electrostatic potential, and the ion flow velocity parallel to the magnetic field direction (the axial direction) [36]. The 


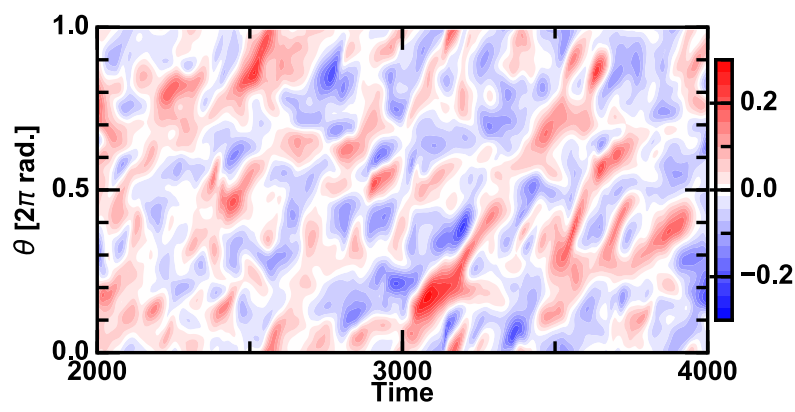

Figure 4. Spatiotemporal evolution of the electron density fluctuation in the Numerical Linear Device (NLD) fluid simulation.

plasma profiles and the turbulence dynamics are self-consistently evolved by the applied sources. The spatially inhomogeneous equilibrium profiles in the electron density, the electrostatic potential, and the parallel flow may drive the resistive drift wave, the Kelvin-Helmholtz instability, and the parallel flow shear driven instability (D'Angelo mode), respectively. The excited waves saturate nonlinearly and also back-interact with the equilibrium profiles, forming a variety of the plasma flow structures [36, 37]. The plasma parameters are given according to the nominal values in the actual cylindrical plasma discharge experiment [38]. In the present case, an azimuthally inhomogeneous parallel momentum source is applied to observe the nonlinear interaction between turbulence and the background flow structure.

Figure 4 shows the spatiotemporal evolution of the electron density fluctuation at the mid-radius of the cylindrical plasma. Turbulence is excited with a large relative amplitude that occasionally shows spatially isolated intermittent bursts. The phase propagation direction seems to be in the positive direction of the azimuthal angle, corresponding to the electron diamagnetic direction. 

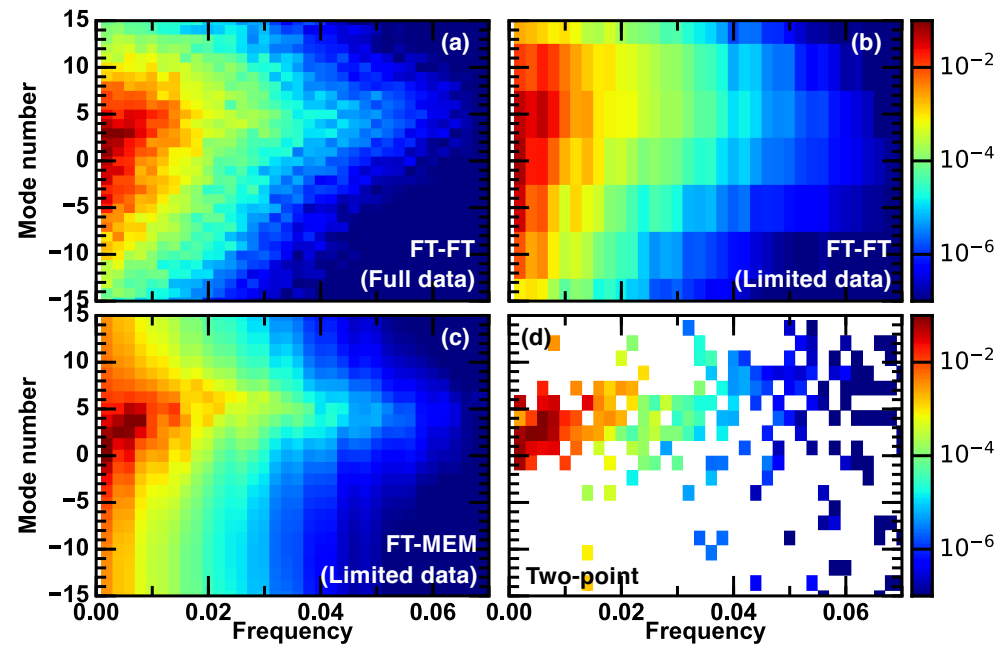

Figure 5. Power spectrum densities of the electron density fluctuation in NLD obtained with various method: (a) FT-FT with the entire azimuthal circumference, (b) FT-FT with $25 \%$ of the azimuthal angle, (c) FT-MEM with $25 \%$ of the azimuthal angle, and (d) two-point statistical method based on FT.

Here, the mode number $m$ is used for describing the spatial scale of the fluctuation instead of the wavenumber $k$. It is defined as $m=k r$, where $r$ is the radius of the cylinder of interest and $m>0$ corresponds to modes with the electron diamagnetic propagation. The frequency-mode number resolved power spectrum densities obtained with various methods are shown in Fig. 5. The original data has a simulation mesh of 128 points in the azimuthal direction. Figure 5 (a) shows the FT-FT power spectrum density using all the azimuthal data points, which corresponds to the true power spectrum density. In order to simulate the experimental situation, where measurement is only performed in a limited spatial domain, eight data points with an interval of four data points are taken. By this data sampling, one fourth of the entire azimuthal circumference is used for estimating the power spectrum density. Figures 5 (b) and (c) show the results of 

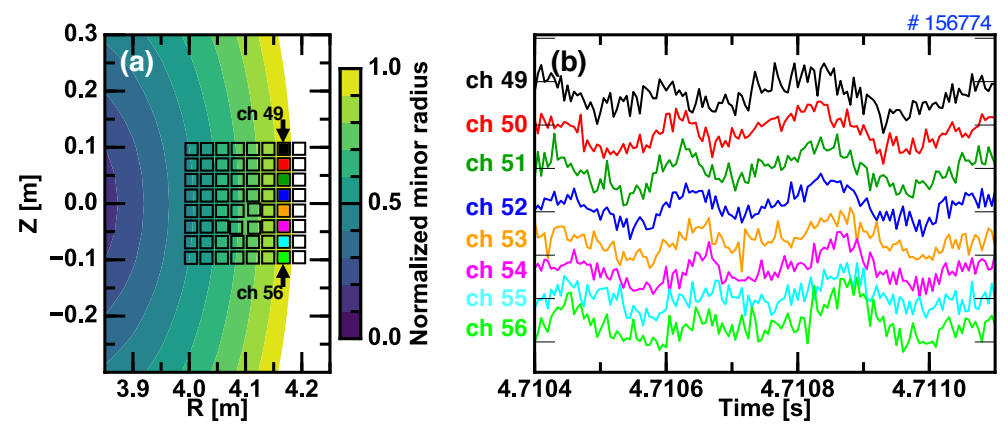

Figure 6. (a) Measurement location and (b) the raw data of the BES in LHD.

the FT-FT method with the Hanning spatial window and the FT-MEM method. Here, $M=1$ is used as the data points for LP for MEM. Although the Akaike FPE criterion suggests $M=2$ as the most appropriate value but with a marginal difference against other values nearby, $M=1$ is chosen to avoid having possible mimic spectral peaks. The FT-MEM method provides a better mode number resolution than the FT-FT method that has less pronounced mode number peaks.

In addition to the FT-FT method and the FT-MEM method, the two-point statistical method based on FT [14] is also applied for comparison. Although the rough features of the spectrum are captured, statistical convergence seems to be insufficient because of the lack of simulation data points particularly for high frequency fluctuation components.

\section{Application II: Experimental data}

The FT-MEM method is applied to an experimental data in the Large Helical Device (LHD). The target data is the poloidal distribution of the electron density fluctuation 


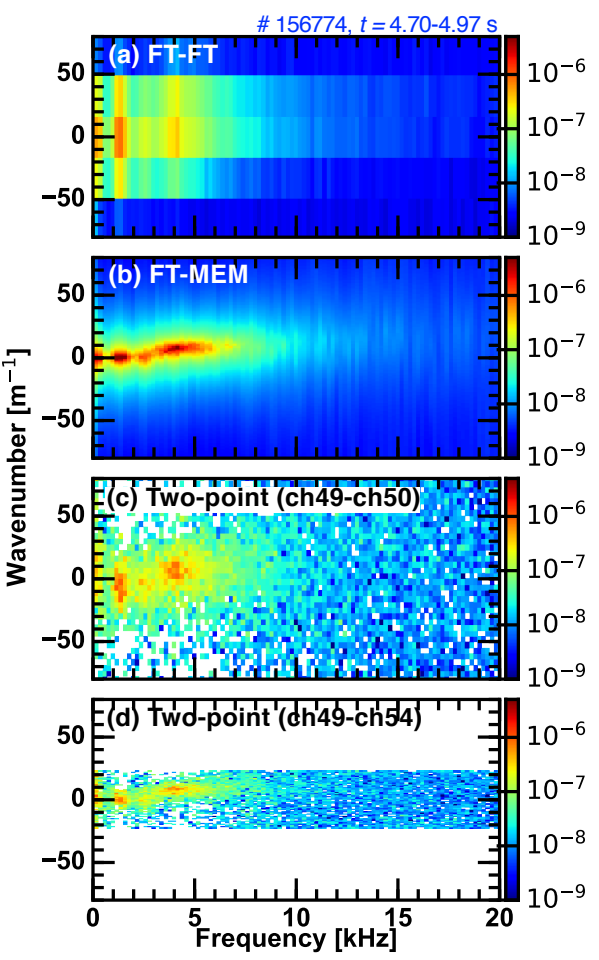

Figure 7. Power spectrum densities of the BES data obtained with various methods: (a) FTFT, (b) FT-MEM, and (c,d) two-point statistical method between ch49-ch50 and ch49-ch54, respectively.

measured by a beam emission spectroscopy (BES) recently installed in LHD [39]. Figure 6 (a) shows the measurement location of the BES in LHD for the shot number of \# 156774. The electron density data are obtained by $8 \times 8$ radial-vertical sight line array with a sampling rate of $200 \mathrm{kHz}$ and an analog bandwidth of $100 \mathrm{kHz}$. One column of the array slightly inside the plasma edge (the second rightmost column) is extracted to examine the poloidal distribution of the fluctuation. Figure 6 (b) is the time evolution of the electron density fluctuation at different vertical positions. The phase of the dominant fluctuating mode propagates from top to bottom, the electron diamagnetic direction. 
The frequency-wavenumber resolved power spectrum densities estimated with different methods are shown in Fig. 7. Positive wavenumber corresponds to modes with the electron diamagnetic propagation. For MEM, $M=1$ is again used. Larger $M$ values were also examined and the results were found to be insensitive to the choice of $M$ except for exaggerated noise peaks in higher frequency range. As is demonstrated for the numerical simulation data, the FT-MEM power spectrum density gives the most sharpest peaks at each frequency. In contrast to the simulation example shown in section 4, the poloidal coverage of the measurement is much smaller fraction, approximately 4 to $5 \%$ of the entire poloidal circumference. Because of this, the wavenumber resolution in the FT-FT method is extremely poor. The two-point statistical method using two adjacent channels (Fig. 7 (c)) also exhibits a less focused wavenumber spectrum, even though the number of ensembles is large enough $(\sim 200$ periods of the dominant frequency components). When the channel interval for the two-point statistical method is increased, the wavenumber resolution becomes finer and the wavenumber spectrum peaks are sharpened as shown by Fig. 7 (d). As a result, the spectral shape resembles to that obtained by the FT-MEM method. However, as the channel interval is increased, the Nyqvist wavenumber is reduced and the observable range of the wavenumber shrinks accordingly. The FT-MEM method reconciles the wide dynamic range and the fine resolution for the wavenumber decomposition. The averaged wavenumbers of the peaks at $f=1-2 \mathrm{kHz}$ and $f=3-5 \mathrm{kHz}$ are $0.6 \mathrm{~m}^{-1}$ and $7.2 \mathrm{~m}^{-1}$, which correspond to the poloidal mode number of $m=0-1$ and $4-5$, respectively. Note that $m=0$ 
may be excluded because a poloidally symmetric MHD mode is not realistic. Unlike the examination of the methods using the numerical simulation, the true spectrum cannot be referred to, therefore, it is impossible to judge the performance of each method in this case. However, considering that the fluctuation is driven by MHD instabilities [39], the power spectrum density with coherent peaks provided by the FT-MEM method seems to the the most plausible result.

\section{Summary}

In this paper, we documented the method for estimating the frequency-wavenumber resolved two-dimensional power spectrum density through combining the Fourier transform and the maximum entropy method. Aiming to improve the wavenumber resolution in a situation, in which available spatial points are severely limited, the maximum entropy method was employed for the spatial structure decomposition. The proposed method was applied to numerical fluid simulation data and experimental data obtained by the beam emission spectroscopy in LHD. Through those examinations, it was found that the proposed method can provide more plausible power spectrum densities than the conventional two-dimensional Fourier transform method or the twopoint statistical method. 


\section{Acknowledgments}

Authors acknowledge T. Ido and T. Morisaki for useful discussion. One of the authors (T. K.) wishes to thank Y. Suzuki and S. Sakakibara for strong support. This work is partly supported by the National Institute for Fusion Science grants (ULHH033) and by the Grant-in-Aid for Scientific Research of JSPS (17K14898).

\section{Appendix}

The python subroutines for obtaining the MEM estimate [26] is shown below. The main routine mcme_k calculates the complex MEM cross spectrum from a multichannel time series of data. The subroutine $f \times 2 f k_{-} k$ performs MEM to the multichannel Fourier components in spacial dimension for each frequency, i.e., the procedure from the second panel to the third panel in Fig. 3. For this spacial MEM, only the auto spectrum calculation is used.

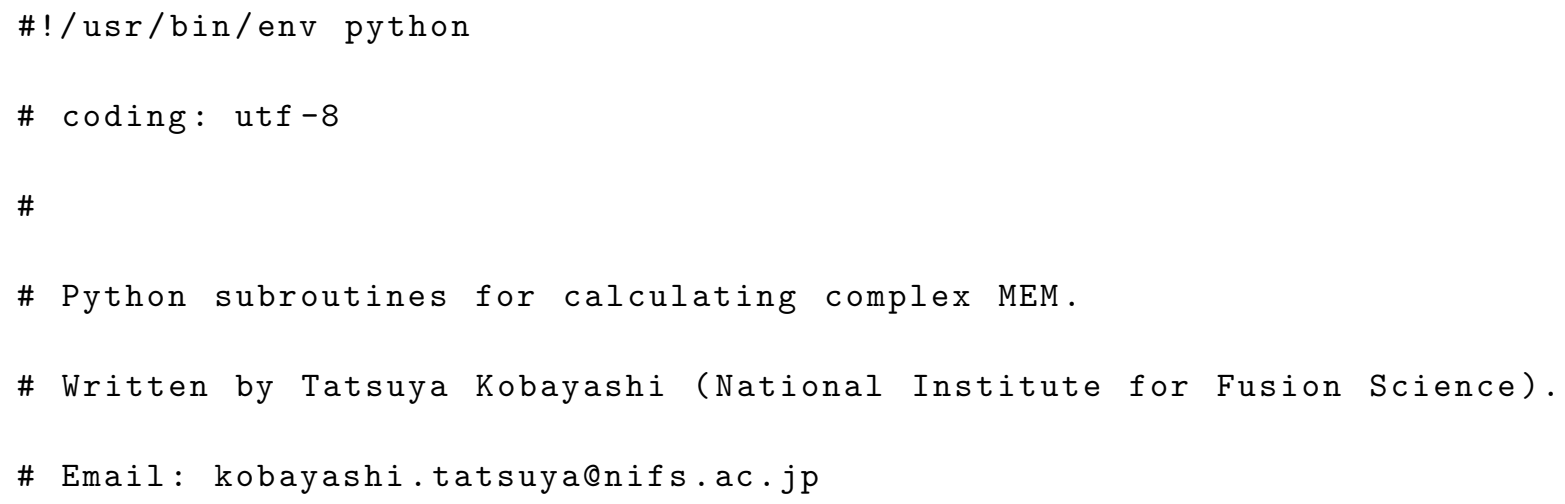




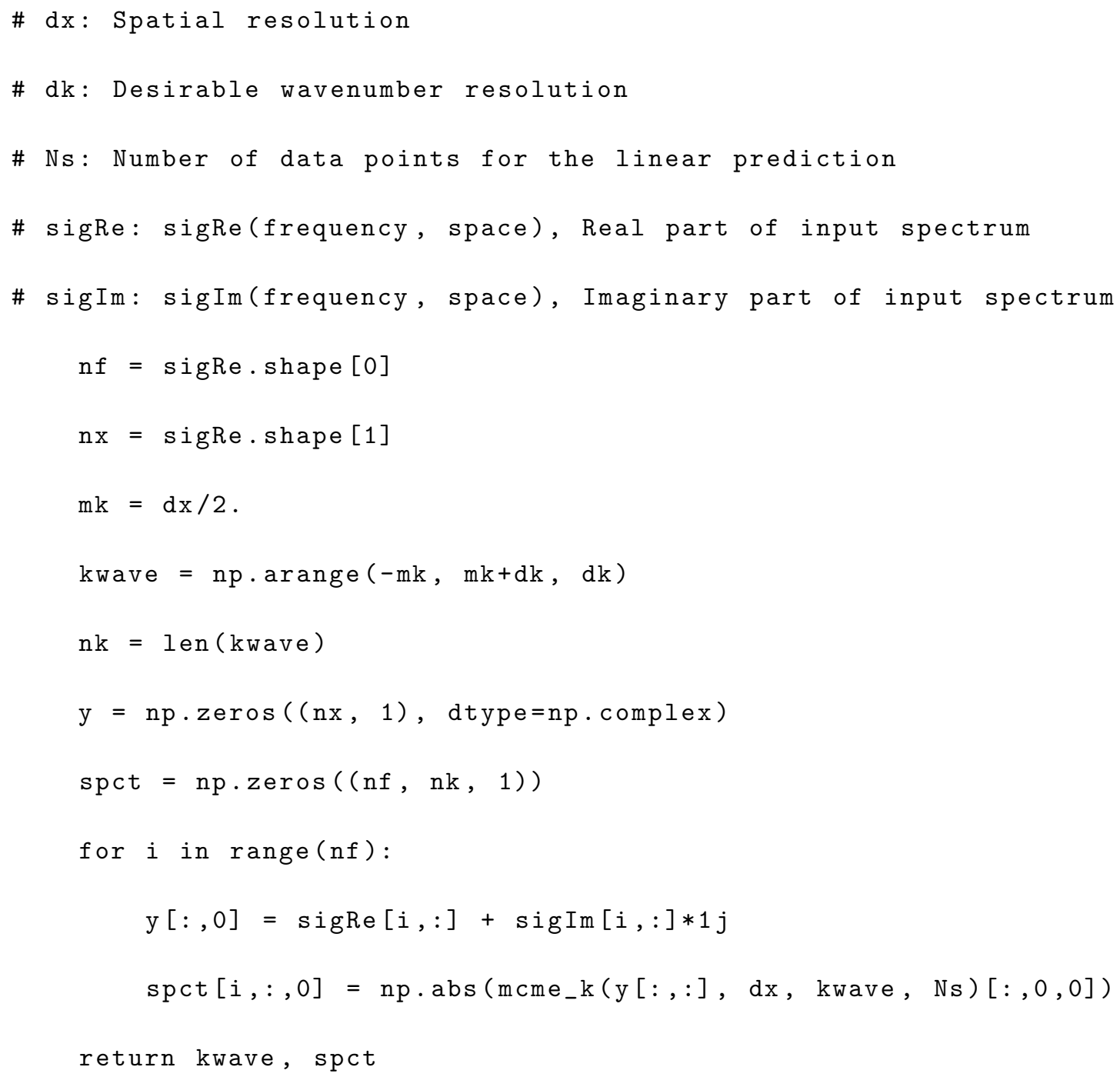


$\mathrm{Nk}=\min (\mathrm{Ns}, \mathrm{Nd}-1)$

spct $=n p \cdot \operatorname{zeros}((d f, p, p)$, dtype $=n p \cdot \operatorname{complex})$

$\mathrm{F}, \mathrm{B}, \mathrm{Pm}, \mathrm{Pd}=\mathrm{mcmecof} \_\mathrm{k}(\mathrm{y}, \mathrm{Ns})$

for $i$ in range (df):

$i F z=n p \cdot l i n a l g \cdot \operatorname{inv}(n p \cdot \operatorname{sum}(F[: N k+1,:,:] * \operatorname{zext}(z[i], N k, p), \operatorname{axis}=0))$ $\operatorname{spct}[i,:,:]=\operatorname{dt} * n p \cdot \operatorname{dot}(n p \cdot \operatorname{dot}(i F z \cdot \operatorname{conjugate}() \cdot T, P m), i F z)$

return spct

def $\operatorname{zext}(z, \mathrm{n}, \mathrm{p})$ :

return $n p . r e p e a t(z * *-n p \cdot \operatorname{arange}(n+1), p * * 2)$. reshape $(n+1, p, p)$

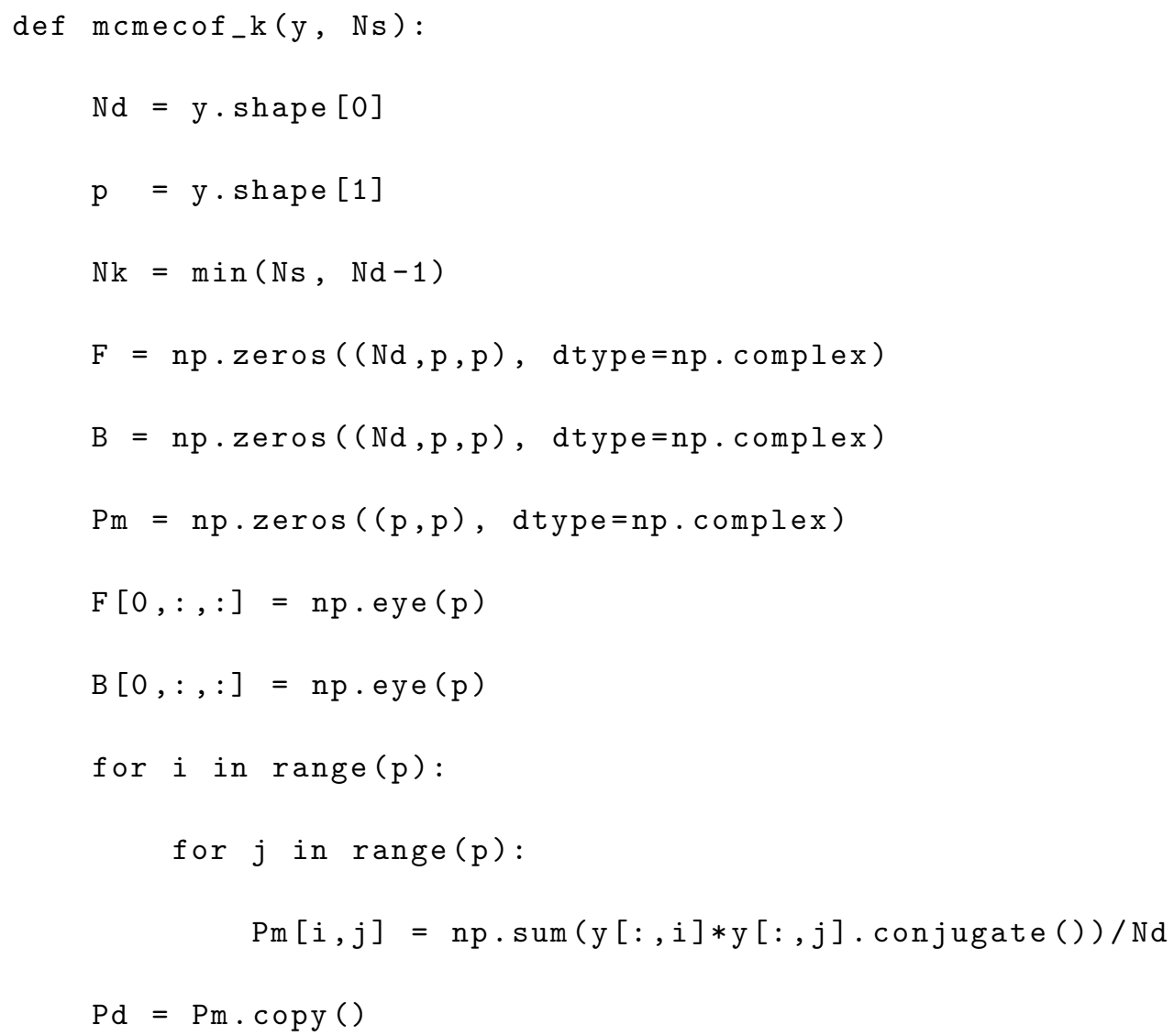


$e r_{-} f=y[1: N d,:]$

er_b $=y[0: N d-1,:]$

for $\mathrm{N}$ in $\mathrm{range}(1, \mathrm{Nk}+1)$ :

$\mathrm{NN}=\mathrm{N}$

er_f, er_b, Pm, Pd, F, B = mcmecof_calc_k (er_f, er $\left.r_{-} b, P m, P d, F, B, N N\right)$

$\operatorname{return}(F, B, P m, P d)$

def mcmecof_calc_k(er_f, er_b, Pm, Pd, F, B, N):

$N d=F$. shape $[0]$

$\mathrm{p}=\mathrm{F}$. shape $[1]$

$\mathrm{M}=\mathrm{Nd}-\mathrm{N}$

$\mathrm{Wm}=1 . / \mathrm{float}(\mathrm{M})$

$F p=n p \cdot z \operatorname{zer}((N d, p, p), \operatorname{dtype}=n p \cdot \operatorname{complex})$

$B p=n p \cdot z \operatorname{zer}((N d, p, p), d t y p e=n p \cdot \operatorname{complex})$

$e_{-} f p=n p \cdot z \operatorname{zeros}((N d-1, p)$, dtype $=n p \cdot \operatorname{complex})$

er_bp $=n p \cdot \operatorname{zeros}((N d-1, p)$, dtype $=n p \cdot \operatorname{complex})$

$E m=n p \cdot z e r o s((p, p)$, dtype $=n p \cdot \operatorname{complex})$

$G m=n p \cdot z e r o s((p, p)$, dtype $=n p \cdot \operatorname{complex})$

$B m=n p \cdot z \operatorname{zeros}((p, p), \operatorname{dtype}=n p \cdot \operatorname{complex})$

$\operatorname{exCOEF}=n p \cdot z \cos ((p * * 2, p * * 2)$, dtype $=n p \cdot \operatorname{complex})$

$C=n p \cdot z \operatorname{zer}(p$, dtype $=n p \cdot \operatorname{complex})$

for $i$ in range $(p)$ :

for $j$ in range $(p)$ : 


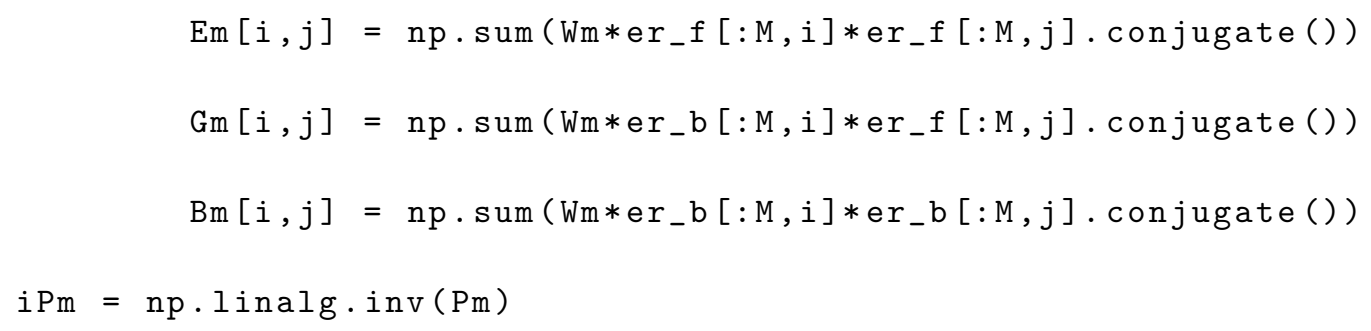




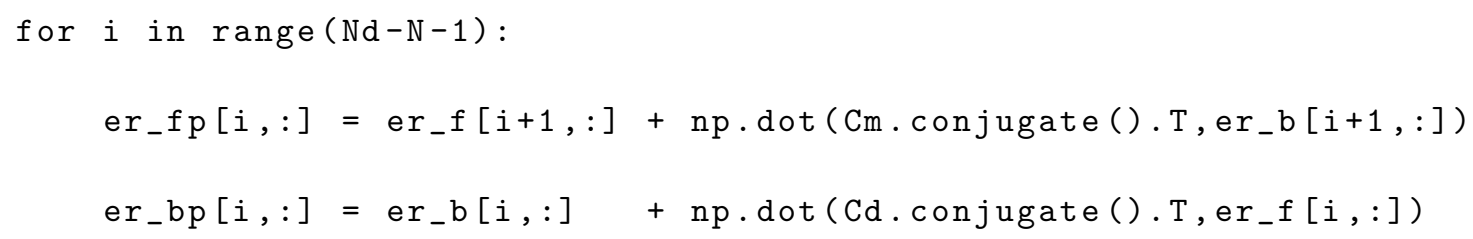




\section{References}

[1] C Schröder, T Klinger, D Block, A Piel, G Bonhomme, and V Naulin 2001 Phys. Rev. Lett. 86 5711

[2] O Grulke and T Klinger 2002 New J. Phys. 467

[3] T Windisch, O Grulke, Volker Naulin, and T Klinger 2011 Plasma Phys. Control. Fusion 53085001

[4] T Yamada, S-I Itoh, T Maruta, N Kasuya, Y Nagashima, S Shinohara, K Terasaka, M Yagi, S Inagaki, Y Kawai, et al 2008 Nat. Phys. 4 721-725

[5] T Kobayashi, S Inagaki, M Sasaki, Y Kosuga, H Arakawa, F Kin, T Yamada, Y Nagashima, N Kasuya, A Fujisawa, et al 2017 Plasma Fusion Res. 121401019

[6] U Stroth, F Greiner, C Lechte, N Mahdizadeh, K Rahbarnia, and M Ramisch 2004 Phys. Plasmas $112558-2564$

[7] B Schmid, P Manz, M Ramisch, and U Stroth 2017 Phys. Rev. Lett. 118055001

[8] A Latten, T Klinger, A Piel, and Th Pierre 1995 Rev. Sci. Instrum. 66 3254-3262

[9] N Krause, C Lechte, J Stöber, U Stroth, E Ascasibar, J Alonso, and S Niedner 2002 Rev. Sci. Instrum. 73 3474-3481

[10] T Yamada, Y Nagashima, S Inagaki, Y Kawai, M Yagi, S-I Itoh, T Maruta, S Shinohara, K Terasaka, M Kawaguchi, et al 2007 Rev. Sci. Instrum. 78123501

[11] S Kado, H Nakatake, K Muraoka, K Kondo, F Sano, T Mizuuchi, S Besshou, H Okada, K Nagasaki, H Funaba, et al 1996 J. Phys. Soc. Jpn. 65 3434-3437

[12] T Ido, Y Miura, K Hoshino, K Kamiya, Y Hamada, A Nishizawa, Y Kawasumi, H Ogawa, Y Nagashima, K Shinohara, et al 2006 Nucl. Fusion 46512

[13] Z Yan, P Gohil, G R McKee, D Eldon, B Grierson, T Rhodes, and C C Petty 2017 Nucl. Fusion 57126015

[14] J M Beall, Y C Kim, and E J Powers 1982 J. Appl. Phys. 53 3933-3940

[15] M VanZeeland, W Gekelman, S Vincena, and J Maggs 2003 Phys. Plasmas 10 1243-1252 
[16] M J Burin, G R Tynan, G Y Antar, N A Crocker, and C Holland 2005 Phys. Plasmas 12052320

[17] P Manz, M Xu, N Fedorczak, S C Thakur, and G R Tynan 2012 Phys. Plasmas 19012309

[18] A Möller 1998 Phys. Plasmas 5 4321-4327

[19] Y Xu, M Van Schoor, R R Weynants, S Jachmich, M Vergote, M W Jakubowski, P Beyer, M Mitri, B Schweer, D Reiser, et al 2007 Nucl. Fusion 471696

[20] A D Liu, T Lan, C X Yu, H L Zhao, L W Yan, W Y Hong, J Q Dong, K J Zhao, J Qian, J Cheng, et al 2009 Phys. Rev. Lett. 103095002

[21] T Kobayashi, Y Nagashima, S Inagaki, H Arakawa, M Sasaki, T Yamada, M Yagi, N Kasuya, A Fujisawa, S-I Itoh, et al 2011 Plasma Fusion Res. 61401050

[22] J P Burg. Maximum entropy spectral analysis. In Proceedings of the 37th Meeting of the Society of Exploration Geophysicists, Oklahoma City, Okla, 1967.

[23] T J Ulrych 1972 J. Geophys. Res. 77 1396-1400

[24] T J Ulrych and T N Bishop 1975 Rev. Geophys. Space Phys. 13 183-200

[25] T J Ulrych and Clayton R W 1976 Phys. Earth Planet. Inter. 12 188-200

[26] O Strand 1977 IEEE Trans. Autom. Control 22 634-640

[27] W H Press, S A Teukolsky, B P Flannery, and W T Vetterling. Numerical recipes in C, chapter 13. Cambridge University Press, 1992.

[28] Y Amagishi, K Saeki, and I J Donnelly 1989 Plasma Phys. Control. fusion 31675

[29] K Tanaka, C A Michael, L N Vyacheslavov, A L Sanin, K Kawahata, T Akiyama, T Tokuzawa, and S Okajima 2008 Rev. Sci. Instrum. 79 10E702

[30] Z Shi, Y Nagayama, S Yamaguchi, D Kuwahara, T Yoshinaga, S Sugito, Y Hirano, H Koguchi, S Kiyama, H Sakakita, et al 2010 Plasma Fusion Res. 5 S1019

[31] K Matsuo, H Iguchi, S Okamura, and K Matsuoka 2012 Rev. Sci. Instrum. 83013501

[32] C A Michael, K Tanaka, L Vyacheslavov, A Sanin, and K Kawahata 2015 Rev. Sci. Instrum. 86 093503

[33] M Ono, K Ida, T Kobayashi, M Yoshinuma, G R McKee, Z Yan, K H Burrell, and X Chen. 
Frequency-Poloidal Wave Number Spectral Analysis of Turbulence in QH-mode plasmas Measured with BES on DIII-D. In 58th Annual Meeting of the APS Division of Plasma Physics, page PP10.074, San Jose, California, 2016.

[34] H Akaike 1970 Ann. Inst. Statist. Math. 22 203-217

[35] N Kasuya, M Yagi, M Azumi, K Itoh, and S-I Itoh 2007 J. Phys. Soc. Jpn. 76044501

[36] M Sasaki, N Kasuya, K Itoh, S Toda, T Yamada, Y Kosuga, Y Nagashima, T Kobayashi, H Arakawa, K Yamasaki, et al 2017 Phys. Plasmas 24112103

[37] M Sasaki, Yann Camenen, A Escarguel, S Inagaki, N Kasuya, K Itoh, and T Kobayashi 2019 Phys. Plasmas 26042305

[38] T Kobayashi, S Inagaki, Y Kosuga, M Sasaki, Y Nagashima, T Yamada, H Arakawa, N Kasuya, A Fujisawa, S-I Itoh, et al 2016 Phys. Plasmas 23102311

[39] T Kobayashi, M Yoshinuma, and K Ida 2020 Plasma Phys. Control. Fusion 62125011 